\title{
ANALISIS DEL PROBLEMA DE ASIGNACION DE FLOTAS FLEXIBLE CON VENTANAS DE TIEMPO DISCRETAS, DURACION VARIABLE, PREFERENCIAS DE SALIDA Y RELACIONES DE PRECEDENCIA. OPTIMIZACION POR COLONIAS DE HORMIGAS
}

\author{
FRANCISCO JAVIER DIEGO MARTIN \\ JOSE ANGEL GONZALEZ MANTECA \\ JAVIER CARRASCO ARIAS \\ DEPARTAMENTO DE INGENIERIA DE ORGANIZACION. ADMINISTRACION DE EMPESASY ESTADISTICA \\ ESCUELA TECNICA SUPERIOR DE INGENIEROS INDUSTRIALES. UNIVERSIDAD POLITECNICA DE MADRID
}

\begin{abstract}
Resumen: Una correcta gestión de flotas incide directamente en la cuenta de resultados de una empresa de transporte de viajeros. Existen variantes del problema de asignación de flotas en cuanto al objetivo que se busca, en cuanto a la forma de modelar el problema y en cuanto a la técnica de resolución. En este trabajo presentamos nuevas restricciones que lo acercan mucho a su aplicación de problemas en empresas reales, para resolverlo a continuación utilizando la optimización mediante colonias de hormigas. Finalmente los resultados obtenidos son comparados con los que se obtendrían operando con la programación lineal, que es el método más utilizado en la resolución de este tipo de problemas.
\end{abstract}

Palabras clave: Asignación de flotas, programación lineal, colonias de hormigas.

\section{Introducción}

Sherali y otros (2005) reflejan la gran importancia en el beneficio que tiene para una compañía aérea la toma de decisiones sobre su flota, y por tanto su correcta gestión constituye un componente esencial para la empresa. Bélanger y otros (2005) definen el problema de asignación de flotas (FAP, Fleet Assignmet Problem) como la asignación del tipo de avión a cada vuelo que ofrezca mayor beneficio global.
La mayoría de los trabajos publicados sobre optimización de flotas están referidos al sector del transporte aéreo; nosotros planteamos un modelo flexible que se pueda aplicar a los sectores aéreo, marítimo y terrestre. Una variable muy considerada en el sector marítimo es la velocidad desarrollada durante el trayecto, ya que el consumo es función de la velocidad, y un pequeño porcentaje de ahorro en el consumo significa cantidades considerables de dinero ahorrado al final del año. En este artículo introducimos como variable la duración del trayecto (y por tanto la velocidad), llevando a optimizaciones con una reducción total de los costes de planificación e, incluso con una reducción del número de vehículos. Hablaremos de trayecto en general como producto que venden las compañías de transporte de viajeros para independizarlo del sector. También hablaremos de estaciones como término general de aeropuerto, estación de autobuses o puerto.

Las ventanas de tiempo en la salida de un trayecto fueron una de las primeras opciones de optimización introducidas, ya que ajustando las horas de salida de los trayectos se puede reducir el número de vehículos necesarios, Bélanger (2004). Esta flexibilidad en la hora de salida aumenta el número de posibles conexiones entre trayectos y ofrece posibilidades de mejorar la calidad de la solución en términos de beneficio o de reducción del número de vehículos. Pero esta flexibilidad debe ser controlada para que no 
se produzcan situaciones no deseables. En este sentido, introducimos el concepto de relaciones de precedencia entre trayectos, como mecanismo de carácter general que permite modelar restricciones de diversa naturaleza que aparecen en los problemas de la vida real, y que sirven para evitar esas situaciones no deseables que puede provocar la apertura de la hora de salida a una ventana de tiempo. Estas relaciones de precedencia se establecen ligando las variables de comienzo y fin de varios trayectos.

Las ventanas de tiempo en la salida ofrecen mucha flexibilidad, pero a menudo no ofrecen la suficiente como para plasmar los requerimientos que se plantean en situaciones de la vida real. Una de estas situaciones son los trayectos que se realizan una o varias veces a la semana. Por ejemplo, el requerimiento puede ser que el trayecto salga el lunes o el martes por la noche, entre las 20:00 y las 23:00. Este requerimiento no se puede expresar mediante una ventana de tiempo en la salida. Nosotros introducimos el concepto de ventana de tiempo discreta en la salida, como un conjunto de ventanas de tiempo que definen la posible salida del trayecto, de forma que la salida del trayecto que fijará el sistema de optimización estará dentro de una sola de las ventanas de tiempo definidas en la salida. Al igual que las relaciones de precedencia, las ventanas de tiempo discretas se presentan como un mecanismo de carácter general que permite modelar muchas situaciones de diferente naturaleza que se presentan en la vida real.

La flexibilidad de las ventanas de tiempo en la salida también puede ser medida en términos de beneficio: podemos dar la flexibilidad a la hora de salida de un trayecto para que se mueva entre las 09:00 y las 1 1:00, pero sabemos que habría algo más de demanda a las 10:00 que a las 09:00 o las 1 1:00. Si se conocieran con exactitud las variaciones de demanda en función de la hora de salida del trayecto, se podría introducir ese beneficio variable en la función objetivo del problema. Pero no suele ser fácil medir esa función y además, la demanda suele ser muy estocástica, por lo que en muchas ocasiones puede ser más práctico aprovechar la experiencia del comercial, y plasmarla en el concepto de hora preferida de salida dentro de la ventana de tiempo de salida del trayecto, que en el ejemplo propuesto al principio de este párrafo sería las 10:00. Introducimos así una variable cualitativa que debe introducida en un modelo matemático cuantitativo, y que nos lleva a un problema multiobjetivo: reducir el número de vehículos, y a igualdad del número de vehículos, maximizar el número de trayectos que salen a su hora preferida.
En este artículo consideramos todos los conceptos introducidos anteriormente para construir un modelo de programación flexible y lo resolvemos aplicando programación lineal y optimización mediante colonias de hormigas, que es una técnica no utilizada hasta hora para este problema.

El artículo está organizado de la siguiente forma: en el apartado 2 abordamos una revisión de las publicaciones sobre el FAP, sus definiciones y técnicas de resolución. En el apartado 3 hacemos una descripción del problema que vamos a tratar. En el apartado 4 mostramos la formulación del problema y en el 5 se describe la resolución del problema mediante las dos técnicas de resolución que hemos utilizado, para finalmente, establecer una comparativa entre ambas técnica en el apartado 6.

\section{Revisión bibliográfica}

loachim (1998) introduce restricciones en las que un mismo vuelo debe salir todos los días para los que esté definido, a la misma hora.Trabaja con diferentes tipos de vehículos, incorpora ventanas de tiempo en la salida y modela el problema mediante programación lineal, que resuelve aplicando un método «branch and bound» diseñado a medida para este problema.

Bélanger (2004) define el FAP como la asignación del tipo de avión que ofrezca mayor beneficio a cada vuelo. El beneficio de un vuelo es función de la demanda prevista de pasajeros y los costes operativos del avión. Considera ventanas de tiempo en la salida, penalizando las salidas de vuelos muy seguidas entre los mismos orígenes y destino. Considera duraciones de trayectos dependientes del tipo de avión. Propone un modelo matemático basado en una estructura de red multi-artículo no lineal. Bélanger (2005) introduce el concepto de homogeneidad para asignar el mismo tipo de avión a los mismos trayectos realizados en diferentes días de la semana para favorecer el servicio de atención de vuelos en tierra.

Sherali (2005) muestra un tutorial muy completo que resalta la integración del FAP con otros procesos de negocio de la empresa, y propone técnicas para resolver el problema, tomando como objetivo el beneficio de la operativa, conocidas las previsiones de pasaje y el coste operativo. Define el FAP como la asignación de tipos de aviones, cada uno con diferentes capacidades, a los vuelos previstos, basada en costes operativos y los potenciales beneficios. Pre- 
senta una serie de modelos que clasifica en primera instancia como modelos básicos (maximiza ingreso menos coste mediante programación lineal entera mixta), modelos integrados (combina el FAP con otros procesos de negocio) y modelos con consideraciones adicionales (pasajeros que realizan varios vuelos seguidos, diferentes vuelos en diferentes días de la semana, travesías).

Suhl (1999) define problemas independientes para cada flota de aviones y considera la ventana de tiempos en la salida como principal opción de optimización para el FAP. El objetivo debería ser maximizar beneficios, pero como es difícil estimar el beneficio de ciertos vuelos en una programación a largo plazo, aproxima ese objetivo por la minimización del número de aviones necesarios. Resuelve el problema mediante programación entera mixta con reducción de coeficientes.

Götz (1999) resuelve el problema de asignación de flotas semanales para compañías aéreas mediante simulación del recocido (SA), mostrando que los resultados son comparables a la programación lineal entera, con la ventaja de que la solución mediante SA requiere menos tiempo de computación y es más fácil modelar restricciones adicionales. Su formulación permite trabajar con varios tipos de vehículos.

La mayor parte de las aplicaciones de optimización mediante colonias de hormigas (ACO) en problemas de flotas están referidas al problema de la generación de rutas de vehículos de distribución de mercancías (Vehicle Routing Problem, VRP). Gambardela (1999) utiliza dos colonias de hormigas que cooperan entre ellas; una colonia minimiza el tamaño de la flota, y la otra minimiza la distancia total recorrida.

No se han encontrado referencias a la resolución del FAP mediante colonias de hormigas. En este trabajo ampliamos el tipo de problemas a los que se aplica esta metaheurística, y planteamos un modelo flexible que permite tanto la resolución del problema que plantea Bélanger (2004) como la del modelo que plantea Suhl (1999), utilizamos como restricción el sistema de penalizaciones que plantea Bélanger, y lo extendemos al concepto más amplio de relación de precedencia que ofrece más capacidades de modelado, incluyendo a las restricciones que plantea loachim (1998). Nuestro modelo da soporte a las ampliamente utilizadas ventanas de tiempo en la salida y las generaliza para ganar en flexibilidad.

\section{Descripción del problema}

El objetivo funcional que nos hemos planteado con este proyecto es permitir modelar el problema de asignación de flotas mediante parámetros generales que permitan plasmar cualquier requerimiento que se plantee en la vida real. Así, generalizamos el concepto de ventana de tiempo en la salida, permitimos duraciones variables de los trayectos para un mismo tipo de vehículo, introducimos las preferencias en la salida, y planteamos relaciones de precedencia entre trayectos. Al problema que incluye todas estas características lo denominamos Problema de Asignación de Flotas Flexible. El objetivo operativo planteado es el de obtener buenas soluciones (óptimas o cercanas a las óptimas) en un tiempo de respuesta aceptable.

Un trayecto viene definido por la siguiente información: un identificador, por ejemplo, HRSTI00, una estación de origen, por ejemplo, AGP (Málaga), una estación de destino, por ejemplo, SCQ (Santiago de Compostela), ventanas de tiempo en la salida, por ejemplo, los lunes entre las 10:00 y las 12:00 o los martes entre las 17:00 y las 18:00, una hora preferida de salida opcional, por ejemplo, los lunes a las | 1:00, y los tipos de vehículo que pueden realizar ese trayecto, por ejemplo, un A320 o un MD87, con una duración de trayecto entre 0 1:30 y 01:45.

Las relaciones de precedencia entre trayectos permiten establecer restricciones entre las horas de salida de dos trayectos. Estas relaciones de precedencia se consideran como una información más de un trayecto. Un mismo trayecto puede tener relaciones de precedencia con varios trayectos a la vez. Estas relaciones pueden ser de dos tipos:

- Distancia entre las salidas: se establece la menor, igual, o mayor distancia entre las salidas de dos trayectos. Por ejemplo, el trayecto HRSTI 30 debe salir al menos dos horas después que el trayecto HRST I00. El motivo que lleve a plantear una de estas relaciones puede ser muy variado: no se dispone de slot, o se debe respetar una frecuencia mínima entre trayectos entre los mismos orígenes y destinos. Se puede fijar un intervalo de separación entre las horas de salida de dos trayectos, fijando una distancia mínima y máxima a la vez: el trayecto HRST200 debe salir al menos tres horas después que el trayecto HRSTI80, pero deben estar separados en menos de cinco horas.

- Misma hora de salida: obliga a que dos trayectos definidos con ventanas de tiempo de salida en días diferentes salgan a la misma hora. Por ejemplo, 
el trayecto HRST250, definido para el martes con una ventana de tiempo en la salida entre las 15:00 y las 17:00 debe salir a la misma hora que el trayecto HRST350, definido para el miércoles con una ventana de salida entre las 15:00 y las 17:00. La causa más común para establecer esta relación es que ambos trayectos corresponden a un mismo producto y se quiere que tenga el mismo horario de salida todos los días.

Con todas estas variables y restricciones se ha conseguido realizar un entorno de programación que ofrece grandes posibilidades de modelado y de optimización al decisor que debe optimizar una flota de vehículos. Se han realizado varios diagramas de Gantt interactivos con altas capacidades gráficas que facilitan mucho el trabajo de optimizar una flota de vehículos. La Figura I muestra el diagrama de Gantt de definición del plan comercial, donde puede verse de forma gráfica toda la información que define cada trayecto y los dos tipos de relaciones de precedencia.

\section{Modelo matemático}

La formulación matemática del Problema de Asignación de Flotas Flexible es la siguiente:

$\operatorname{Min} \sum_{t}^{T_{i}} \sum_{v}^{V_{i}} c^{t} X_{0 i v}^{t}+\sum_{t}^{T_{i}} \sum_{v}^{V_{i}} \sum_{j}^{T_{j}} \sum_{w}^{V_{j}} c_{i j}^{t} X_{i v, w j}^{t}$

$\operatorname{Max} \sum_{i} P_{i}$

Sujeto a las siguientes restricciones:

$$
\sum_{t}^{T_{i}} \sum_{v}^{V_{i}} X_{i v 0}^{t}+\sum_{t}^{T_{i}} \sum_{v}^{V_{i}} \sum_{j}^{T_{j}} \sum_{w}^{V_{j}} X_{i v, w j}^{t}=1 \quad \forall i l \quad i \neq j, \quad X_{i v}^{t} \rightarrow X_{i w}^{t}
$$

$$
\begin{aligned}
& \sum_{t}^{T_{i}} \sum_{v}^{V_{i}} X_{0 i v}^{t}+\sum_{t}^{T_{j}} \sum_{v}^{V_{i}} \sum_{j}^{T_{j}} \sum_{w}^{V_{j}} X_{j w, v}^{t}=l \quad \forall i l \quad i \neq j, \quad X_{j w}^{t} \rightarrow X_{i v}^{t} \\
& C_{j w}-\left(F_{i v}-e_{i j}\right) \geq M_{i v}\left(X_{i, j w}^{t}-I\right) \quad \forall i, v, w, t l \quad i \neq j, \quad X_{i v}^{t} \rightarrow X_{j w}^{t} \\
& \sum_{v}^{V_{i}} \gamma_{i v}=|\forall i| V_{i}>1 \\
& X_{j w, i v}^{t} \leq \gamma_{i v} \quad \forall i, t, j, w / \quad i \neq j, \quad V_{i}>l, \quad v \in\left\{0, \ldots, V_{i-1}\right\}, \quad X_{i v}^{t} \rightarrow X_{j w}^{t} \\
& C_{i v} \leq \gamma_{i v} C_{i v}^{m a} \quad \forall i l \quad V_{i}>l, \quad v \in\left\{0, \ldots, V_{i-1}\right\} \\
& C_{i v} \geq \gamma_{i v} C_{i v}^{m i} \quad \forall i l \quad V_{i}>l, \quad v \in\left\{0, \ldots, V_{i-1}\right\} \\
& F_{i v}-C_{i v} \geq d_{i, m i}^{t} \quad v \in\left\{0, \ldots, v_{i-1}\right\} \\
& F_{i v}-C_{i v} \leq d_{i, m a}^{t} \quad v \in\left\{0, \ldots, v_{i-1}\right\} \\
& \sum_{i}^{T_{i}} \sum_{v}^{V_{i}} X_{i v, j w}^{t}=\sum \sum X_{j w, k x}^{t} \quad \forall j, w, t, \quad X_{i v}^{t} \rightarrow X_{j w}^{t}, X_{j w}^{t} \rightarrow X_{k x}^{t} \\
& C_{j}-C_{i} \geq D_{j i} \quad \forall i, j / R_{i j} \in \Re \\
& C_{j}-C_{i} \leq D_{j i} \quad \forall i, j / R_{i j} \in \Re \\
& C j-C i=D_{j i} \quad \forall i, j / R_{i j} \in \Re \\
& C_{i}<C P_{i} \oplus C_{i}>C P_{i} \oplus P_{i}=1
\end{aligned}
$$

Donde:

Los índices $i, j \in\{0, \ldots, n-1\}$ son los $n$ trayectos que entran en el proceso de dimensionado, $v, w \in$ $\left\{0, \ldots, V_{i}-1\right\}$ son las $V_{i}$ ventanas de tiempo en la salida del trayecto $i, t \in\{0, \ldots, T-I\}$ son los $T_{i}$ tipos de vehículo que pueden realizar el trayecto i. Las siguientes son variables binarias de decisión: $X_{i v 0}^{t} \in\{0,1\}$

Figura I

Diagrama de Gantt interactivo para la definición del plan comercial

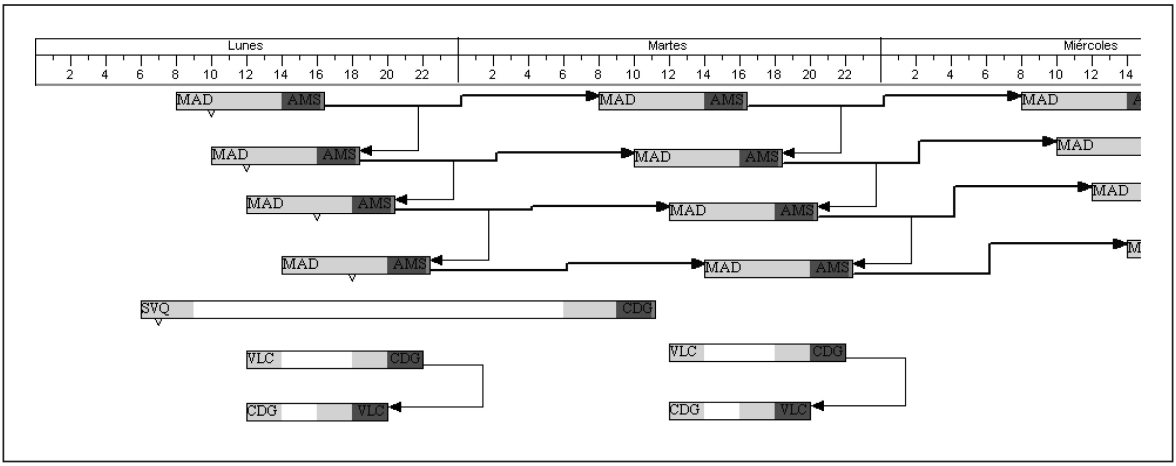


indica si la ventana $\vee$ del trayecto i es el último trayecto de una rotación, $X_{\text {Oiv }}^{t} \in\{0,1\}$ indica si la ventana $\vee$ del trayecto i es el primer trayecto de una rotación, $X_{i v, j w}^{t} \in\{0,1\}$ indica si a la ventana $\vee$ del trayecto i le sigue inmediatamente en una rotación la ventana j del trayecto $w$ con el tipo de vehículo $t$, $\gamma_{i v}$ indica si se asigna la ventana $\vee$ del trayecto $i, y P_{i}$ $\in\{0,1\}$ indica si el trayecto i comienza en su hora de salida preferida. Las siguientes son variables de decisión continuas: $C_{i v}$ representa la hora de salida del trayecto i cuando comienza dentro de la ventana de tiempo en la salida $v, F_{\text {iv }}$ representa la hora de llegada del trayecto i cuando comienza dentro de la ventana de tiempo en la salida v. Los siguientes son valores constantes: $c_{t}$ representa el coeficiente de coste en que se incurre al utilizar un vehículo del tipo $t, c_{i j}^{t}$ representa el coeficiente de coste en que se incurre cuando un vehículo de tipo t realiza los trayectos i y j seguidos dentro de una rotación, $e_{i j}$ es el tiempo de escala mínimo que ha de respetarse cuando al trayecto i le sigue inmediatamente el trayecto j en la misma rotación, $C_{i v}^{m i}$ es la mínima hora de salida de la ventana de salida $\vee$ del trayecto i, $C_{i v}^{m a}$ es la máxima hora de salida de la ventana de salida $\vee$ del trayecto i, $M_{i v}$ es el margen de comienzo de la ventana de salida $\vee$ del trayecto i, $C P_{i}$ es la hora de salida preferida para el trayecto i, di,ma es la máxima duración del trayecto i cuando lo realiza un vehículo de tipo t, $d_{i, m i}^{t}$ es la mínima duración del trayecto i cuando lo realiza un vehículo de tipo $t, R_{i j}$ representa una relación de precedencia entre trayectos, y $\mathfrak{R}$ es el conjunto de relaciones de precedencia.

Las restricciones [1] y [2] aseguran que todos los trayectos están dentro de alguna rotación. Esto se consigue asegurando que desde todas las ventanas y para todos los tipos de vehículos de un determinado trayecto i solo se puede ir a un trayecto (ecuación [I]), y que hasta todas las ventanas y para todos los tipos de vehículos de un trayecto i solo se llega desde un trayecto (ecuación [2]). La restricción [3] asegura que dos trayectos con ventanas de tiempo no se solapan y por tanto solo se crean rotaciones con secuencias de trayectos físicamente realizables. Las restricciones [4], [5], [6] y [7] aseguran que solo se asigna un trayecto dentro de una de las ventanas de tiempo definidas para el trayecto y que el comienzo del trayecto está dentro de esa ventana de tiempo en la salida. Las restricciones [3] relacionan unos trayectos con otros, de forma que un trayecto no pueda comenzar hasta que termina el que le precede. Las restricciones [4] aseguran que la salida de un trayecto se realizará dentro de su ventana de tiempo. Las restricciones [8] y [9] aseguran que la duración del trayecto está dentro de los márgenes estableci- dos por tipo de vehículo. La restricción [10] es una restricción de continuidad que asegura que la ventana de un trayecto a la que se llega desde otro trayecto es la misma que conecta con el siguiente trayecto. Las restricciones [ I I], [ I2] y [13] modelan las relaciones de precedencia entre trayectos.

La restricción [14] es una restricción lógica que establece una relación entre la variable binaria $P_{i}$ (que indica que un trayecto sale en su hora preferida) con el comienzo del trayecto i, que es una variable completamente introducida en el modelo. La restricción lógica debe ser transformada a forma lineal para su posterior resolución mediante programación lineal: partimos de $C_{i} \geq C P_{i}$, se define $S_{l i}=\left(C_{i}-C P_{i}\right) / M_{i}$, así $S_{1 i} \leq 1$. Se define $X_{1 i} \in\{0,1\} / X_{1 i} \geq S_{\mid i}$ y tenemos que si $C_{i}>C P_{i}$ entonces $X_{1 i}=1$. Ahora partimos de $C_{i} \leq C P_{i}$, se define $S_{2 i}=\left(C P_{i}-C_{i}\right) / M_{i}$, así $S_{2 i} \leq 1$. Se define $X_{2 i} \in\{0,1\} / X_{2 i} \geq S_{2 i}$ y tenemos que si $C_{i}<C P_{i}$ entonces $X_{2 i}=1$. Si pasamos la restricción [14] a ecuación lineal tenemos $X_{1 i}+X_{2 i}+P_{i}=1$.

La función objetivo se ha expresado de forma general en función de unos coeficientes de costes que dependen del tipo de vehículo. Si se quiere minimizar el número de vehículos totales a utilizar se deben considerar únicamente los coeficientes $c^{t}$ igualándolos a uno, y considerando $c_{i j}^{t}=0$. Si se quiere que la decisión acerca del número de vehículos de cada tipo a considerar sea función de un coste fijo de utilización de cada tipo de vehículo, se deben considerar $c^{t}$ y hacer $c_{i j}^{t}=0$. Si la decisión sobre el número de vehículos a calcular se quiere hacer función de un coste fijo de utilización y función de los trayectos asignados a cada tipo de vehículo, se deben considerar $c^{t} y$ hacer $c_{i j}^{t}$. Si se conocen los beneficios que tiene la asignación de cada trayecto a cada uno de los tipos de vehículos que pueden realizarlo, se puede utilizar la siguiente función objetivo para maximizar el beneficio, donde $b_{i j}^{t}$ y $b_{i 0}^{t}$ es el beneficio del trayecto i al ser asignado al tipo de vehículo t:

$$
\operatorname{Max} \sum_{t}^{T_{i}} \sum_{v}^{V_{i}} \sum_{j}^{T_{j}} \sum_{w}^{V_{j}} b_{i j}^{t} X_{i v, w j}^{t}+\sum_{t}^{T_{i}} \sum_{v}^{V_{i}} b_{i}^{t} X_{i v 0}^{t}-\sum_{t}^{T_{i}} \sum_{v}^{V_{i}} c^{t} X_{0 i v}^{t}
$$

Con toda esta formulación podemos plantear dos problemas:

I. Sin preferencia de salida:

$$
\operatorname{Min} \sum_{t}^{T_{i}} \sum_{v}^{V_{i}} c^{t} X_{0 i v}^{t}+\sum_{t}^{T_{i}} \sum_{v}^{V_{i}} \sum_{j}^{T_{j}} \sum_{w}^{V_{j}} c_{i j}^{t} X_{i v, w j}^{t}
$$


Sujeto a:

Restricciones [I] [2]...[13]

2. Con preferencia de salida:

a) $C=\operatorname{Min} \sum_{t}^{T_{i}} \sum_{v}^{V_{i}} c^{t} X_{0 i v}^{t}+\sum_{t}^{T_{i}} \sum_{v}^{V_{i}} \sum_{j}^{T_{j}} \sum_{w}^{V_{j}} c_{i j}^{t} X_{i v, w j}^{t}$

Sujeto a:

Restricciones [I] [2]...[13]

b) $\operatorname{Max} \sum_{i} P_{i}$

Sujeto a:

$$
\sum_{t}^{T_{i}} \sum_{v}^{V_{i}} c^{t} X_{0 i v}^{t}+\sum_{t}^{T_{i}} \sum_{v}^{V_{i}} \sum_{j}^{T_{j}} \sum_{w}^{V_{j}} c_{i j}^{t} X_{i v, w j}^{t} \leq C
$$

Restricciones [ I ] [2]... [ 3]

$$
\begin{aligned}
& S_{l i}=\frac{C_{i}-C P_{i}}{M_{i}} \\
& X_{1 i} \geq S_{1 i} \\
& S_{2 i}=\frac{C P_{i}-C_{i}}{M_{i}} \\
& X_{2 i} \geq S_{2 i} \\
& X_{l i}+X_{2 i}+P_{i}=1
\end{aligned}
$$

\section{Donde:}

$X_{1 i} \in\{0,1\}$ y $X_{2 i} \in\{0,1\}$ son variables binarias de apoyo a la inclusión de las preferencias en la salida como variables de optimización, mientras que $S_{1 i}$ y $S_{2 i}$ son variables continuas de apoyo a la inclusión de las preferencias en la salida como variables de optimización.

\section{Resolución del problema}

\section{I. Programación lineal}

La gran ventaja de la programación lineal reside en que al ser un método exacto garantiza que la solución encontrada es la solución óptima. Para la reso- lución del problema se ha utilizado la librería de programación GLPK v8.4, Markorin (2006).

\subsection{Colonias de hormigas}

La optimización por colonias de hormigas (Ant Colony Optimization, ACO) fue introducida por Colorni, Dorigo y Maniezzo ( 199 |) como una metaheurística para resolver problemas de optimización combinatoria. La observación de las hormigas en búsqueda de comida fue la inspiración para la resolución de los problemas de optimización. Las hormigas se comunican entre ellas sobre las fuentes de comida mediante una esencia aromática llamada feromona. Marcan el camino por el que pasaron con una cantidad de feromona que depende de la longitud del camino que lleva a la comida y de la calidad de ésta. Otras hormigas observan las estelas de feromona, que les sirve como guía hacia las mejores fuentes de comida. Este comportamiento de las hormigas sirve para resolver problemas de optimización combinatoria mediante simulación: las hormigas artificiales recorren el espacio de soluciones simulando a las hormigas reales recorriendo el entorno, los valores de la función objetivo de las soluciones simulan la calidad de las fuentes de comida, y la memoria adaptativa corresponde a las estelas de feromona.

La optimización por colonias de hormigas (ACO) requiere que el problema sea representado mediante un grafo por cuyos nodos deben ir pasando las hormigas artificiales en el proceso de construcción de soluciones. Dada la naturaleza del problema de dimensionado de flotas, la representación como grafo es muy sencilla si consideramos cada trayecto como los nodos y la transición entre trayectos como las ramas del grafo.

A grandes rasgos, un algoritmo por colonización de hormigas consta de tres procesos que se repiten a lo largo de una serie de iteraciones: construir soluciones, evaluar soluciones, y depositar feromonas. En función de cómo se realicen esos pasos, existen unas cuantas variantes de algoritmo. Tras numerosas pruebas hemos seleccionado el algoritmo ACS (Ant Colony System) como el más eficiente para nuestro problema. A continuación detallamos los pasos seguidos para la resolución del FAP flexible:

Construcción de soluciones: En cada iteración se construyen m soluciones en paralelo. En cada solución, las rutas de los vehículos se construyen haciendo que las hormigas vayan de trayecto en trayecto desde un 
nodo origen ficticio hasta que no puede visitar más trayectos. Cuando una hormiga k está en un trayecto i, debe elegir el siguiente trayecto j de forma probabilista dentro del conjunto de trayectos factibles $\mathrm{N}_{i}^{k}$ (es decir, el conjunto de trayectos que conectan con el trayecto i, y que todavía no han sido visitados). La regla probabilista usada para construir una ruta es la siguiente: con probabilidad qo se selecciona el trayecto con mayor $\left[\tau_{i j}\right]^{\alpha}\left[\eta_{i j}\right]^{\beta}$, y con probabilidad $\left(I-q_{0}\right)$ se selecciona el nodo j con una probabilidad proporcional a $\left[\tau_{i j}\right]^{\alpha}\left[\eta_{i j}\right]^{\beta}, j \in N_{i}^{k}$ según la ecuación $[\mathrm{I}]$

$$
P_{i j}= \begin{cases}\frac{\left[\tau_{i j}\right]^{\alpha} \cdot\left[\eta_{i j}\right]^{\beta}}{\sum_{i \in N_{i}^{k}}\left[\tau_{i j}\right]^{\alpha} \cdot\left[\eta_{i j}\right]^{\beta}} \quad \text { si } \quad j \in \mathrm{N}_{i}^{k} \\ 0 \quad \text { en otro caso }\end{cases}
$$

donde $\alpha, \beta$, y qo son parámetros: $\alpha$ representa la importancia que se da a las estelas de feromona , $\beta$ representa la importancia de la información heurística $\eta_{i j}$, y $q_{0}$ determina la importancia relativa entre explotación y exploración: cuanto más pequeño sea qo, mayor será probabilidad de usar la regla probabilista descrita en la ecuación [I].Tras numerosas pruebas, hemos determinado los siguientes valores de estos parámetros: $\alpha=1, \beta=2$, y $q_{0}=0,8$

Depósito de feromona: En ACS la actualización de las estelas de feromona se realiza de forma local y global. La actualización local se realiza durante la construcción de soluciones, mientras que la global se realiza tras la fase de construcción de soluciones. En esta fase global solo se utiliza la mejor solución encontrada hasta el momento. La actualización global se realiza de la siguiente forma:

$$
\tau_{i j}=(\mid-\rho) \cdot \tau_{i j}+\rho / \int_{\psi}^{g b} \quad \forall(i, j) \in \psi^{g h}
$$

donde $\rho(0 \leq \rho \leq 1)$ es la tasa de evaporación de feromonas y $J_{\Psi}^{g h}$ es el valor objetivo de $\Psi^{g b}$, que es la mejor solución encontrada hasta el momento. El valor que se ha utilizado para $\rho$ es 0,1 . La actualización local de feromona se realiza de la siguiente forma:

$$
\tau_{i j}=(\mid-\rho) \cdot \tau_{i j}+\rho \cdot \tau_{0}
$$

donde $\tau_{0}$ es el valor inicial de las estelas de feromona, que se calcula como $\tau_{0}=1 /\left(n \cdot \int_{\Psi}^{h}\right)$, donde $n$ es el número de trayectos y $\int_{\Psi}^{h}$ es el valor objetivo de la solución inicial generada por una heurística FIFO (se selecciona el vehículo que antes haya llegado a la estación).

\section{Resultados}

Se han creado tres juegos de datos de diferente tamaño: el A con 20 trayectos, el B con 106 trayectos, y el $C$ con 412 trayectos, y se han realizado cuatro tipos de pruebas: todos los trayectos sin ventanas de tiempo y con ellas, ambos resueltos mediante programación lineal y ACO. En los problemas sin ventanas de tiempo, la programación lineal se muestra muy eficiente y es preferible frente a ACO ya que ofrece la solución óptima. En problemas con ventanas de tiempo, solo para el problema $A$ es eficiente la programación lineal, pero con los problemas B y C la programación lineal aumenta mucho el tiempo de cálculo y $\mathrm{ACO}$ se vuelve más efectivo, pero perdemos esa seguridad en haber encontrado la mejor solución. En el problema A, ACO encuentra la mejor solución, y en los problemas B y C, se obtienen valores muy cercanos a la mejor solución, incluso llegándose a alcanzar en algunas ocasiones.

\section{Referencias}

BELANGER, N.; DESAULNIERS, G.; SOUMIS, F., y DESROISERS, J. (2004). Periodic airline fleet assignment problem with time windows, spacing contraints, and time dependent revenues. European Journal of Operational Research.

BELANGER, N.; DESAULNIERS, G.; SOUMIS, F.; DESROISERS, J., y LAVIGNE, J. (2005). Weekly airline fleet assignment with homogeneity.Transportation Research, Part B.

COLORNI, A.; DORIGO, M., y MANIEZZO,V. (|99|). Distributed optimization by Ant Colonies. In F.Varela, and P. Bourgine, editors, Proc. Europ. Conf. Artificial Life (ECAL'9I), pages 134- |42, Elsevier Publishing.

GAMBARDELA, L. M.; TAILLARD, É., y AGAZZI, G. ( 1999). MACS-VRPTW: A multiple ant colony system for vehicle routing problems with time windows. IDSIA, Lugano, Switzerland.

GOTZ, S.; GROTHKLGAS, S.; KLIEVER, G., yTSCHOKE, S. (1999). Solving the Weekly Fleet Assignment Problem for Large Airlines. MIC'99. III Metaheuristics International Conference

IOACHIM, I.; DESROSIERS, J.; SOUMIS, F., y BELANGER, N. (1998). Fleet assignment and routing with schedule synchronization constraints. European journal of Operational Research.

MARKORIN, A. (2005). GLPK: GNU Lineal Programming Kit. Disponible en www.gnu.org. 
MOUDANI,W., y MORA-CAMINO, F.A dynamic approach for aircraft assignment and maintenance scheduling by airlines. LAAS du CNRS, ENAC, Air Transport Department, Toulouse, France SHERALI, H. D.; BISH, E. K., y ZHU, X. (2005). Airline fleet assignment concepts, models and algo- rithms. Grado Department od Industrial ans Systems Engineering, Virginia Polytechnic Insitute, USA

SUHL, U., y SUHL, L. M. ( 1999). Solving Airline-fleet Sheduling Problems with Mixed-integer Programming. Macmillan Press. 